\title{
Code Obfuscation Against Abstract Model Checking Attacks
}

\author{
Roberto Bruni ${ }^{1}$, Roberto Giacobazzi ${ }^{2,3}$, and Roberta Gori ${ }^{1}$ \\ 1 Dipartimento di Informatica, Università di Pisa, Italy \\ 2 Dipartimento di Informatica, Università di Verona, Italy \\ 3 IMDEA SW Institute, Spain
}

\begin{abstract}
Code protection technologies require anti reverse engineering transformations to obfuscate programs in such a way that tools and methods for program analysis become ineffective. We introduce the concept of model deformation inducing an effective code obfuscation against attacks performed by abstract model checking. This means complicating the model in such a way a high number of spurious traces are generated in any formal verification of the property to disclose about the system under attack. We transform the program model in order to make the removal of spurious counterexamples by abstraction refinement maximally inefficient. A measure of the quality of the obfuscation obtained by model deformation is given together with a corresponding best obfuscation strategy for abstract model checking based on partition refinement.
\end{abstract}

\section{Introduction}

Software systems are a strategic asset, which in addition to correctness deserves security and protection. This is particularly critical with the growth of mobile computing, where the traditional black-box security model, with the attacker not able to see into the implementation system, is not anymore adequate. Code protection technologies are increasing their relevance due to the ubiquitous nature of modern untrusted environments where code runs. From home networks to consumer devices (e.g., mobile devices, cloud, and IoT devices), the running environment cannot guarantee integrity and privacy. Existing techniques for software protection originated with the need to protect license-checking code, particularly in games or in IP protection. Sophisticated techniques, such as white-box (WB) cryptography and software watermarking, were developed to prevent adversaries from circumventing anti-piracy protection in digital rights management systems.

A WB attack model to a software system $\mathcal{S}$ assumes that the attacker has full access to all of the components of $\mathcal{S}$, i.e., $\mathcal{S}$ can be inspected, analysed, verified, reverse-engineered, or modified. The goal of the attack is to disclose properties of the run-time behaviour of $\mathcal{S}$. These can be a hidden watermark [22 1819], a cryptographic key or an invariance property for disclosing program semantics and make correct reverse engineering [7]. Note that standard encryption is only partially applicable for protecting $\mathcal{S}$ in this scenario: The transformed code has to be executable while being protected. Protection is therefore implemented 
as obfuscation [6. Essentially, an obfuscator is a compiler that transforms a program $p$ into a semantically equivalent one $\mathcal{O}(p)$ but harder to analyse and reverse engineer. In many cases it is enough to guarantee that the attacker cannot disclose the information within a bounded amount of time and with limited resources available. This is the case if new releases of the program are issued frequently or if the information to be disclosed is some secret key whose validity is limited in time, e.g., when used in pay-per-view mobile entertainment and in streaming of live events. Here the goal of the code obfuscation is to prevent the attacker from disclosing some keys before the end of the event.

The current state of the art in code protection by obfuscation is characterised by a scattered set of methods and commercial/open-source techniques employing often ad hoc transformations; see [7 for a comprehensive survey. Examples of obfuscating transformations include code flattening to remove control-flow graph structures, randomised block execution to inhibit control-flow graph reconstruction by dynamic analysis, and data splitting to obfuscate data structures. While all these techniques can be combined together to protect the code from several models of attack, it is worth noting that each obfuscation strategy is designed to protect the code from one particular kind of attack. However, as most of these techniques are empirical, the major challenges in code protecting transformations are: (1) the design of provably correct transformations that do not inject flaws when protecting code, and (2) the ability to prove that a certain obfuscation strategy is more effective than another w.r.t. some given attack model.

In this paper we consider a quite general model of attack, propose a measure to compare different obfuscations and define a best obfuscation strategy.

The aim of any attack is to disclose some program property. It is known that many data-flow analyses can be cast to model checking of safety formulas. For example, computing the results of a set of data-flow equations is equivalent to computing a set of states that satisfies a given modal/temporal logic specification 20]21. Even if several interesting properties are not directly expressed as safety properties, because they are existentially quantified over paths, their complements are relevant as well and are indeed safety properties, i.e. they are requested to hold for all reachable states. For these reasons $\forall C \mathrm{CL}^{*}$ is a suitable formal logic to express those program properties the attacker wants to disclose.

In this context, program analysis is therefore the model checking of a $\forall \mathrm{CTL}^{*}$ formula on a(n approximate) model associated with the program. The complexity of software analysis requires automated methods and tools for WB attack to code. Since the attacker aims to disclose the property within a bounded time and using bounded resources, approximate methods such as abstract interpretation [8] or abstract model checking [3] are useful to cope with the complexity of the problem. The abstraction here is helpful to reduce the size of the model keeping only the relevant information which is necessary for the analysis. Safety properties expressed in $\forall C T L^{*}$ can be model-checked using abstraction refinement techniques (CEGAR [2]) as in Fig. 1. An initial (overapproximated) abstraction of the program is model-checked against the property $\phi$. If the verification proves that $\phi$ holds true, then it is disclosed. Similarly, if an abstract 


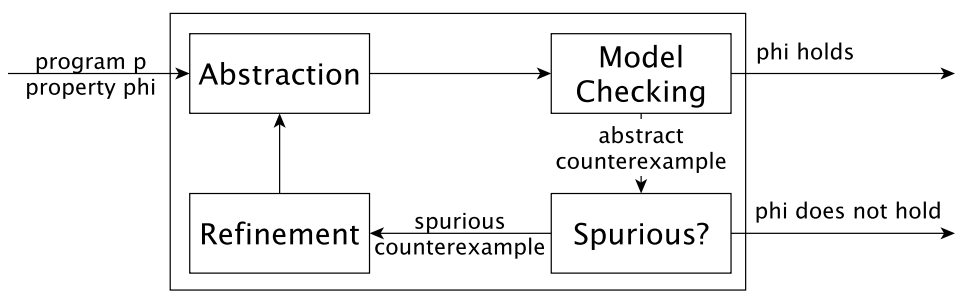

Fig. 1. Counterexample guided abstraction refinement (CEGAR) framework

counterexample is found that corresponds to a concrete counterexample, it is disclosed that $\phi$ is not valid. An abstract counterexample that is present in the existential overapproximation but not in the concrete model is called spurious. If a spurious counterexample is found the abstraction is refined to eliminate it and the verification is repeated on the refined abstraction. Of course, the coarser the abstraction that can be used to verify the property the more effective the attack is. Indeed, the worst case for the attacker is when the verification cycle must be repeated until the refined abstraction coincides with the concrete model.

Given a program $p$ and a $\forall \mathrm{CTL}^{*}$ property $\phi$ to be obfuscated we aim to:

1. define a measure to compare the effectiveness of different obfuscations of $p$; 2. derive an optimal (w.r.t. the above measure) obfuscation of $p$.

The measure of obfuscation that we propose is based on the size of the abstract model that allows the attacker to disclose the validity of $\phi$. Intuitively, the larger the size of the model, the more resources and computation power the attacker needs to spend to reach the goal.

We propose a systematic model deformation that induces a systematic transformation on the program (obfuscation). The idea is to transform the source program in such a way that:

1. its semantics is preserved: the model of the original program is isomorphic to the (reachable) part of the model of the obfuscated program (Theorem 1);

2. the performance is preserved;

3. the property $\phi$ is preserved by the transformation (Theorem 21;

4. such transformation forces the CEGAR framework to ultimately refine the abstract model of the transformed program into the concrete model of the original program (Theorem 3). Therefore any abstraction-based model checking becomes totally ineffective on the obfuscated program.

CEGAR can be viewed as a learning procedure, devoted to learn the partition (abstraction) which provides a (bisimulation) state equivalence. Our transformation makes this procedure extremely inefficient. Note that several instances of the CEGAR framework are possible depending on the chosen abstraction and refinement techniques (e.g. predicate refinement, partition refinement) and that CEGAR can be used in synergy with other techniques for compact representation of the state space (e.g., BDD) and for approximating the best refined 
abstraction (e.g., SAT solvers). Notably, CEGAR is employed in state-of-the-art tools as Microsoft's SLAM engine for the SDV framework 17. Here we focus on the original formulation of CEGAR based on partition refinement, but we believe that our technique can be extended to all the other settings.

As many obfuscating transformations, our method relies on the concept of opaque expressions and opaque predicate that are expressions whose value is difficult for the attacker to figure out. Opaque predicates and expressions are formulas whose value is uniquely determined (i.e. a constant), independently from the parameters they receive, but this is not immediately evident from the way in which the formula is written. For example it can be proved that the formula $x^{2}-34 y^{2} \neq 1$ is always true for any integer values of $x$ and $y$. Analogously, the formula $\left(x^{2}+x\right) \bmod 2 \neq 0$ is always false. These expression/predicate are, in general, constructed using number properties that are hard for an adversary to evaluate. Of course such predicates can be parameterised so that each instance will look slightly different. Opaque predicates/expressions are often used to inject dead code in the obfuscated program in such a way that program analysis cannot just discard it. For example, if the guard $x^{2}-34 y^{2} \neq 1$ is used in a conditional statement, then the program analysis should consider both the "then" and the "else" branches, while only the first is actually executable. In this paper: i) opaque expressions will be used to hide from the attacker the initial values of the new variables introduced by our obfuscation procedure; and ii) opaque predicates will be used to add some form of nondeterminism originated from model deformations. The effects of the opaque expressions and predicates will be similar: since the attacker will not be able to figure out their actual values, all the possible values have to be taken into account.

Plan of the paper. In Section 2 we recall CEGAR and fix the notation. In Section 3 we introduce the concept of model deformation and define the measure of obfuscation. In Section 4 we define a best obfuscation strategy. Our main results are in Section 5 . Some concluding remarks are in Section 6

Related works. With respect to previous approaches to code obfuscation, all aimed to defeat attacks based on specific abstractions, we define the first transformation that defeats the refinement strategy, making our approach independent on the specific attack carried out by abstract model checking.

Most existing works dealing with practical code obfuscation are motivated by either empirical evaluation or by showing how specific models of attack are defeated, e.g., decompilation, program analysis, tracing, debugging (see [7]). Along these lines, 23 firstly considered the problem of defeating specific and well identified attacks, in this case control-flow structures. More recently 1 shows how suitable transformations may defeat symbolic execution attacks. We follow a similar approach in defeating abstract model-checking attacks by making abstraction refinements maximally inefficient. The advantage in our case is in the fact that we consider abstraction refinements as targets of our code protecting transformations. This allows us both to extract suitable metrics and to apply our transformations to all model checking-based attacks. 
A first attempt to formalise in a unique framework a variety of models of attack has been done in 13 10/14 in terms of abstract interpretation. The idea is that, given an attack implemented as an abstract interpreter, a transformation is derived that makes the corresponding abstract interpreter incomplete, namely returning the highest possible number of false alarms. The use of abstract interpretation has the advantage of making it possible to include in the attack model the whole variety of program analysis tools. While this approach provides methods for understanding and comparing qualitatively existing obfuscations with respect to specific attacks defined as abstract interpreters, none of these approaches considers transformations that defeat the abstraction refinement, namely the procedure that allows to improve the attack towards a full disclosure of the obfuscated program properties.

Even if the relation between false alarms and spurious counterexamples is known [15 to the best of our knowledge, no obfuscation methods have been developed in the context of formal verification by abstract model checking, or more in general by exploiting structural properties of computational models and their logic.

\section{Setting The Context}

\subsection{Abstract Model Checking}

Given a set Prop of propositions, we consider the fragment $\forall \mathrm{CTL}^{*}$ of the temporal logic $\mathrm{CTL}^{*}$ over Prop $4[12$. Models are Kripke structures $\mathcal{K}=\langle\Sigma, R, I,\|\cdot\|\rangle$ with a transition system $\langle\Sigma, R\rangle$ having states in $\Sigma$ and transitions $R \subseteq \Sigma \times \Sigma$, initial states $I \subseteq \Sigma$, and an interpretation function $\|\cdot\|$ : Prop $\longrightarrow \wp(\Sigma)$ that maps each proposition $\mathrm{p}$ to the set $\|\mathrm{p}\| \subseteq \Sigma$ of all and only states where $\mathrm{p}$ holds. For $\forall \mathrm{CTL}^{*}$ the notion of satisfaction of a formula $\varphi$ in $\mathcal{K}$ is as usual [11, written $\mathcal{K} \models \varphi$ A path in $\langle\Sigma, R, I,\|\cdot\|\rangle$ is an infinite sequence $\pi=\left\{s_{i}\right\}_{i \in \mathbb{N}}$ of states such that $s_{0} \in I$ and for every $i \in \mathbb{N}, R\left(s_{i}, s_{i+1}\right)$. Terminating executions are paths where the final state repeats forever. We will sometimes use $\pi$ to denote also a finite path prefix $\left\{s_{i}\right\}_{i \in[0, n]}$ for some $n \in \mathbb{N}$. Given a path $\pi=\left\{s_{i}\right\}_{i \in \mathbb{N}}$ and a state $x \in \Sigma$, we write $x \in \pi$ if $\exists i \in \mathbb{N}$ such that $x=s_{i}$.

Any state partition $P \subseteq \wp(\Sigma)$ defines an abstraction merging states into abstract states, i.e., an abstract state is a set of concrete states and the abstraction function $\alpha_{P}: \Sigma \rightarrow \wp(\Sigma)$ maps each state $s$ into the partition class $\alpha_{P}(s) \in P$ that contains $s$. The abstraction function can be lifted to a pair of adjoint functions $\alpha_{P}: \wp(\Sigma) \rightarrow \wp(\Sigma)$ and $\gamma_{P}: \wp(\Sigma) \rightarrow \wp(\Sigma)$, such that for any $X \in \wp(\Sigma)$, $\alpha_{P}(X)=\bigcup_{x \in X} P(x)$ [19. When the partition $P$ is clear from the context we omit the subscript. A partition $P$ with abstraction function $\alpha$ induces an abstract Kripke structure $\mathcal{K}_{P}=\left\langle\Sigma^{\sharp}, R^{\sharp}, I^{\sharp},\|\cdot\|^{\sharp}\right\rangle$ that has abstract states in $\Sigma^{\sharp}=P$, ranged by $s^{\sharp}$, and is defined as the existential abstraction induced by $P$ :

$-R^{\sharp}\left(s_{1}^{\sharp}, s_{2}^{\sharp}\right)$ iff $\exists s, t \in \Sigma . R(s, t) \wedge \alpha(s)=s_{1}^{\sharp} \wedge \alpha(t)=s_{2}^{\sharp}$,

$-s^{\sharp} \in I^{\sharp}$ iff $\exists t \in I . \alpha(t)=s^{\sharp}$,

- $\|\mathrm{p}\| \stackrel{\sharp \text { def }}{=}\left\{s^{\sharp} \in \Sigma^{\sharp} \mid s^{\sharp} \subseteq\|\mathrm{p}\|\right\}$, 
An abstract path in the abstract Kripke structure $\mathcal{K}_{P}$ is denoted by $\pi^{\sharp}=$ $\left\{s_{i}^{\sharp}\right\}_{i \in \mathbb{N}}$. The abstract path associated with the concrete path $\pi=\left\{s_{i}\right\}_{i \in \mathbb{N}}$ is the sequence $\alpha(\pi)=\left\{\alpha\left(s_{i}\right)\right\}_{i \in \mathbb{N}}$. Vice versa, we denote by $\gamma\left(\pi^{\sharp}\right)$ the set of concrete paths whose abstract path is $\pi^{\sharp}$, i.e., $\gamma\left(\pi^{\sharp}\right)=\left\{\pi \mid \alpha(\pi)=\pi^{\sharp}\right\}$.

A counterexample for $\varphi$ is either a finite abstract path or a finite abstract path followed by a loop. Abstract model checking is sound by construction: If there is a concrete counterexample for $\varphi$ then there is also an abstract counterexample for $i t$. Spurious counterexamples may happen: If there is an abstract counterexample for $\varphi$ then there may or may not be a concrete counterexample for $\varphi$.

\subsection{Counter-Example Guided Abstraction Refinement}

With an abstract Kripke structure $\mathcal{K}^{\sharp}$ and a formula $\varphi$, the CEGAR algorithm works as follows [2]. $\mathcal{K}^{\sharp}$ is model checked against the formula. If no counterexample to $\mathcal{K}^{\sharp} \models \varphi$ is found, the formula $\varphi$ is satisfied and we conclude. If a counterexample $\pi^{\sharp}$ is found which is not spurious, i.e., $\gamma\left(\pi^{\sharp}\right) \neq \emptyset$, then we have an underlying concrete counterexample and we conclude that $\varphi$ is not satisfied. If the counterexample is spurious, i.e., $\gamma\left(\pi^{\sharp}\right)=\emptyset$, then $\mathcal{K}^{\sharp}$ is further refined and the procedure is repeated. The procedure illustrated in Fig. 1 induces an abstract model-checker attacker that can be specified as follows in pseudocode.

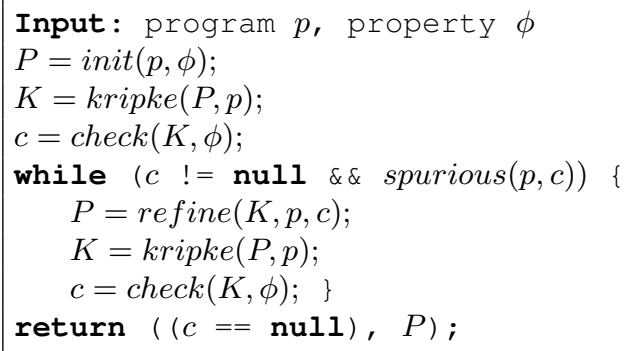

Here we denote by init a function that takes a program $p$ and the property $\varphi$ and returns an initial abstraction $P$ (a partition of variable domains); a function kripke that generates the abstract Kripke structure associated with a program $p$ and the partition $P$; a function check that takes an abstract Kripke structure $K$ and a property $\varphi$ and returns either null, if $K \models \varphi$, or a (deterministically chosen) counterexample $c$; a predicate spurious that takes the program $p$ and an abstract counterexample $c$ and returns true if $c$ is a spurious counterexample and false otherwise; and a function refine $(K, p, c)$ that returns a partition refinement so to eliminate the spurious counterexample $c$. As the model is finite, the number of partitions that refine the initial partition is also finite and the algorithm terminates by returning a pair: a boolean that states the validity of the formula, and the final partition that allows to prove it.

If several spurious counterexamples exist, then the selection of one instead of another may influence the refinements that are performed. For example, the same refinement that eliminates a spurious counterexample may cause the disappearance of several other ones. However, all the spurious counterexamples must 
be eliminated. When we assume that check is deterministic, we just fix a total order on the way counterexamples are found. For example, we may assume that a total order on states is given (e.g., lexicographic) and extend it to paths.

Central in CEGAR is partition refinement. The algorithm identifies the shortest prefix $\left\{s_{i}^{\#}\right\}_{i \in[0, k+1]}$ of the abstract counterexample $\pi^{\sharp}$ that does not correspond to a concrete path in the model. The second to last abstract state $s_{k}^{\#}$ in the prefix, called a failure state, is further partitioned by refining the equivalence classes in such a way that the spurious counterexample is removed. To refine $s_{k}^{\#}$, the algorithm classifies the concrete states $s \in s_{k}^{\#}$ in three classes:

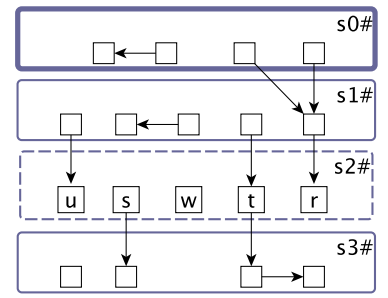

Fig. 2. Dead, bad and irrelevant states

- Dead states: they are reachable states $s \in s_{k}^{\#}$ along the spurious counterexample prefix but they have no outgoing transitions to the next states in the spurious counterexample, i.e., there is some concrete path prefix $\pi \in \gamma\left(\left\{s_{i}^{\#}\right\}_{i \in[0, k]}\right)$ such that $s \in \pi$ and for any $s^{\prime} \in s_{k+1}^{\#}$ it holds $\neg R\left(s, s^{\prime}\right)$.

- Bad states: they are non-reachable states $s \in s_{k}^{\#}$ along the spurious counterexample prefix but have outgoing transitions that cause the spurious counterexample, i.e., for any concrete path prefix $\pi \in \gamma\left(\left\{s_{i}^{\#}\right\}_{i \in[0, k]}\right)$ we have $s \notin \pi$, but $R\left(s, s^{\prime}\right)$ for some concrete state $s^{\prime} \in s_{k+1}^{\#}$.

- Irrelevant states: they are neither dead nor bad, i.e., they are not reachable and have no outgoing transitions to the next states in the counterexample.

Example 1 (Dead, bad and irrelevant states). Consider the abstract path prefix $\left\{s_{0}^{\#}, s_{1}^{\#}, s_{2}^{\#}, s_{3}^{\#}\right\}$ in Fig. 2. Each abstract state is represented as a set of concrete states (the smaller squares). The arrows are the transitions of the concrete Kripke structure and they induce abstract arcs in the obvious way. We use a thicker borderline to mark $s_{0}^{\#}$ as an initial abstract state and a dashed borderline to mark $s_{2}^{\#}$ as a failure state. The only dead state in $s_{2}^{\#}$ is $r$, because it can be reached via a concrete path from an initial state but there is no outgoing transition to a state in $s_{3}^{\#}$. The states $s$ and $t$ are bad, because they are not reachable from initial states, but have outgoing transitions to states in $s_{3}^{\#}$. The states $u$ and $w$ are irrelevant.

CEGAR looks for the coarsest partition that separates bad states from dead states. The partition is obtained by refining the partition associated with each variable. The chosen refinement is not local to the failure state, but it applies globally to all states: It defines a new abstract Kripke structure for which the spurious counterexample is no longer valid. Finding the coarsest partition corresponds to keeping the size of the new abstract Kripke structure as small as possible. This is known to be a NP-hard problem [2], due to the combinatorial 
explosion in the number of ways in which irrelevant states can be combined with dead or bad states. In practice, CEGAR applies a heuristic: irrelevant states are not combined with dead states. The opposite option of separating bad states from both dead and irrelevant states is also viable.

In the following we assume that states in $\Sigma$ are defined as assignments of values to a finite set of variables $x_{1}, \ldots, x_{n}$ that can take values in finite domains $D_{1}, \ldots, D_{n}$. Partitions over states are induced by partitions on domains. A partition $P$ of variables $x_{1}, \ldots, x_{n}$ is a function that sends each $x_{i}$ to a partition $P_{i}=P\left(x_{i}\right) \subseteq \wp\left(D_{i}\right)$. Given the abstractions associated with partitions $P_{1}, \ldots, P_{n}$ of the domains $D_{1}, \ldots, D_{n}$, the states of the abstract Kripke structure are defined by the possible ranges of values that are assigned to each variable according to the corresponding partition.

\subsection{Programs}

We let $\mathcal{P}$ be the set of programs written in the syntax of guarded commands [5] (e.g., in the style of CSP, Occam, XC), according to the grammar below:

$$
\begin{array}{ll|c|c|c}
d::=x \in D \mid d, d & g::=x \in V & \text { true } & g \wedge g|g \vee g| \neg g \\
a::=x=e \mid a, a & c::=g \Rightarrow a & |c| c & p::=(d ; g ; c)
\end{array}
$$

where $x$ is a variable, $V \subseteq \bigcup_{i} D_{i}$ is a finite set of values, and $e$ is a well-defined expression over variables. A declaration is a non-empty list of basic declarations $x \in D$ assigning a domain $D$ to the variable $x$. We assume that all the variables appearing in a declaration $d$ are distinct. A basic guard is a membership predicate $x \in V$ or true. A guard $g$ is a formula of propositional logic over basic guards. We write $x \notin V$ for $\neg(x \in V)$. An action is a non-empty list of assignments. A single assignment $x=e$ evaluates the expression $e$ in the current state and updates $x$ accordingly. If multiple assignments $x_{1}=e_{1}, \ldots, x_{k}=e_{k}$ are present, the expressions $e_{1}, \ldots, e_{k}$ are evaluated in the current state and their values are assigned to the respective variables. All the variables appearing in the left-hand side of multiple assignments must be distinct, so the order of assignments is not relevant. A basic command consists of a guarded command $g \Rightarrow a$ : it checks if the guard $g$ is satisfied by the current state, in which case it executes the action $a$ to update the state. Commands can be composed in parallel: any guarded command whose guard is satisfied by the current state can be applied. A program $(d ; g ; c)$ consists of a declaration $d$, an initialisation proposition $g$ and a command $c$, where all the variables in $g$ and $c$ are declared in $d$.

Example 2 (A sample program). We consider the following running example program (in pseudocode) that computes in $y$ the square of the initial value of $x$.

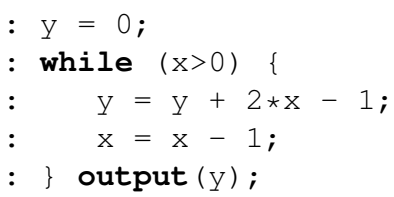




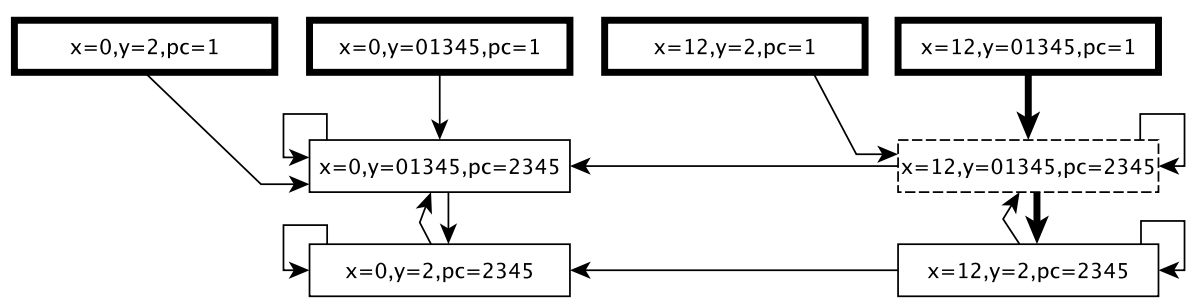

Fig. 3. An abstract Kripke structure

For simplicity we assume the possible values assigned to variables are in quite limited ranges, but starting with larger sets of values would not change the outcome of the application of the CEGAR algorithm. The translation of the previous program in the syntax of guarded commands is the program $p=$ $\left(d ; g ; c_{1}\left|c_{2 a}\right| c_{2 b}\left|c_{3}\right| c_{4}\right)$, written below in CSP-like syntax. Intuitively, it is obtained by adding an explicit variable $p c$ for the program-counter and then encoding each line of the source code as a basic command.

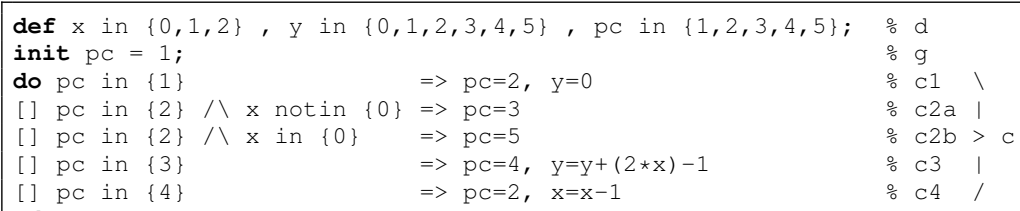

In this context, an attacker may want to check if $y$ is ever assigned the value 2 , which can be expressed as the property: $\phi \stackrel{\text { def }}{=} \forall G(p c \in\{1\} \vee y \notin\{2\})$ (i.e. for all paths, for all states in the path it is never the case that $p c \neq 1$ and $y=2$ ).

Let $d=\left(x_{1} \in D_{1}, \ldots, x_{n} \in D_{n}\right)$. A state $s=\left(x_{1}=v_{1}, \ldots, x_{n}=v_{n}\right)$ of the program $(d ; g ; c)$ is an assignment of values to all variables in $d$, such that for all $i \in[1, n]$ we have $v_{i} \in D_{i}$ and we write $s(x)$ for the value assigned to $x$ in $s$. Given $c=\left(g_{1} \Rightarrow a_{1}|\cdots| g_{k} \Rightarrow a_{k}\right)$, we write $s \models g_{j}$ if the guard $g_{j}$ holds in $s$ and $s\left[a_{j}\right]$ for the state obtained by updating $s$ with the assignment $a_{j}$.

The concrete Kripke structure $\mathcal{K}(p)=\langle\Sigma, R, I,\|\cdot\|\rangle$ associated with $p=$ $(d ; g ; c)$ is defined as follows: the set of states $\Sigma$ is the set of all states of the program; the set of transitions $R$ is the set of all and only $\operatorname{arcs}\left(s, s^{\prime}\right)$ such that there is a guarded command $g_{j} \Rightarrow a_{j}$ in $c$ with $s \models g_{j}$ and $s^{\prime}=s\left[a_{j}\right]$; the set of initial states $I$ is the set of all and only states that satisfy the guard $g$; the set of propositions is the set of all sentences of the form $x_{i} \in V$ where $i \in[1, n]$ and $V \subseteq D_{i}$; the interpretation function is such that $\left\|x_{i} \in V\right\|=\left\{s \mid s\left(x_{i}\right) \in V\right\}$.

Example 3 (A step of CEGAR). The Kripke structure associated with the program $p$ from Example 2 has 90 states, one for each possible combination of values for its variables $x, y, p c$. There are 18 initial states: those where $p c=1$.

Assume that the attacker, in order to prove $\phi$, starts with the following initial partition (see [5]):

$$
x:\{\{0\},\{1,2\}\} \quad y:\{\{2\},\{0,1,3,4,5\}\} \quad p c:\{\{1\},\{2,3,4,5\}\}
$$




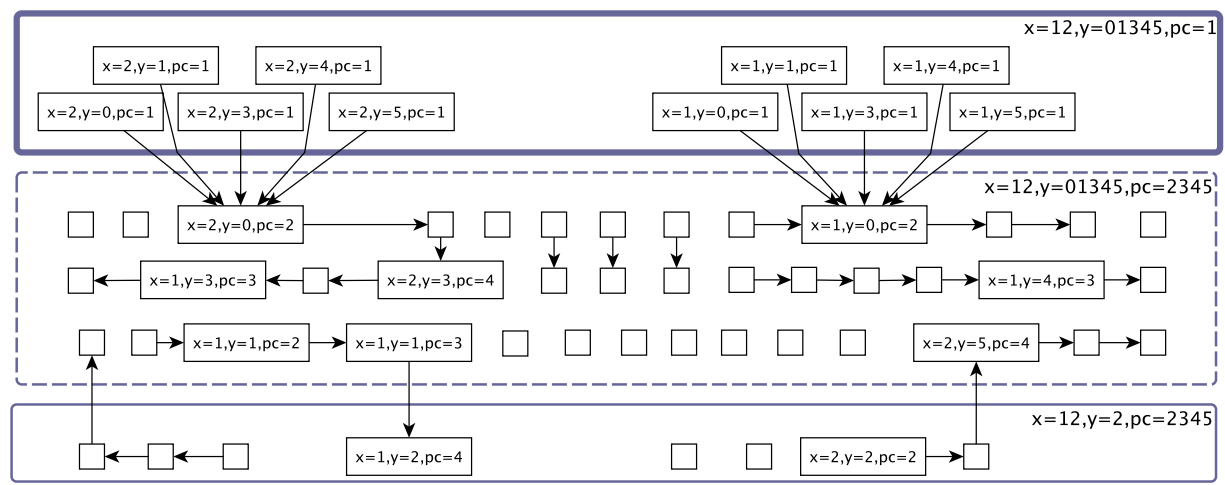

Fig. 4. Failure state

The corresponding abstract Kripke structure has just 8 states (see Fig. 3 with 4 initial states marked with bold borderline in Fig. 3, where, to improve readability, we write, e.g., $y=01345$ instead of the more verbose $y \in\{0,1,3,4,5\}$.

There are several paths that lead to counterexamples for $\phi$. One such path is the one marked with bold arrows in Fig. 3. It is detailed in Fig. 4 by showing the underlying concrete states. It is a spurious counterexample, because there is no underlying concrete path. The abstract failure state is $(x \in\{1,2\}, y \in$ $\{0,1,3,4,5\}, p c \in\{2,3,4,5\})$, depicted with dashed borderline in Fig. 3 . It contains one bad concrete state $(x=1, y=1, p c=3)$, two dead states $((x=1, y=$ $0, p c=2)$ and $(x=2, y=0, p c=2))$ and 37 irrelevant states.

By partition refinement we get the following refined partition:

$$
x:\{\{0\},\{1,2\}\} \quad y:\{\{2\},\{0\},\{1,3,4,5\}\} \quad p c:\{\{1\},\{2\},\{3,4,5\}\}
$$

Thus the corresponding abstract Kripke structure has now 18 states, six of which are initial states. While the previously considered spurious counterexample has been removed, another one can be found and, therefore, CEGAR must be repeated, (see Fig. 6 discussed in Example 5 for further steps).

\section{Model Deformations}

We introduce a systematic model deformation making abstract model checking harder. The idea is to transform the Kripke structure, by adding states and transitions in a conservative way. We want to somehow preserve the semantics of the model, while making the abstract model checking less efficient, in the sense that only trivial (identical) partitions can be used to prove the property. In other words, any non-trivial abstraction induces at least one spurious counterexample.

Let $\mathbb{M}$ be the domain of all models specified as Kripke structures. Formally, a model deformation is a mapping between Kripke structures $\mathfrak{D}: \mathbb{M} \rightarrow \mathbb{M}$ such that for a given formula $\phi$ and $\mathcal{K} \in \mathbb{M}: \mathcal{K}=\phi \Rightarrow \mathfrak{D}(\mathcal{K}) \models \phi$ and there exists a partition $P$ such that $\mathcal{K}_{P}=\phi \Rightarrow \mathfrak{D}\left(\mathcal{K}_{P}\right) \not \models \phi$. In this case we say that $\mathfrak{D}$ 


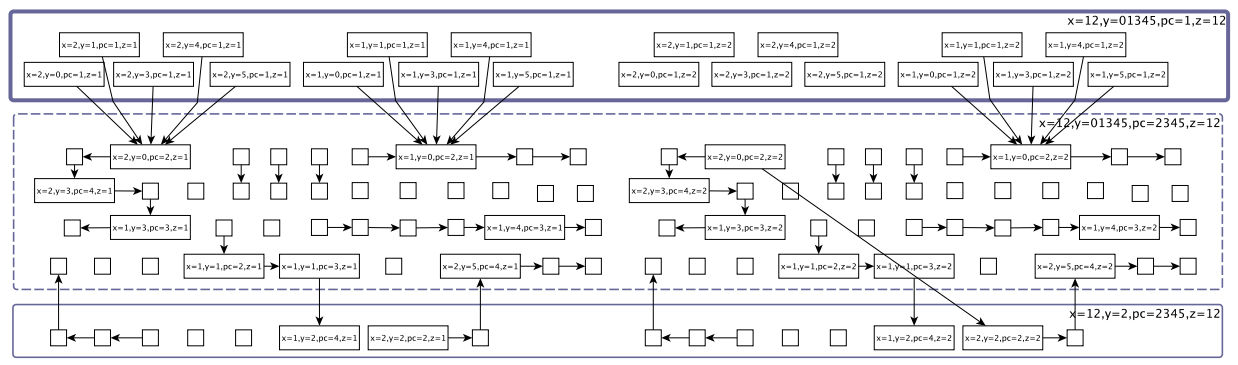

Fig. 5. A detail of a deformation

is a deformation for the partition $P$. Thus, a model deformation makes abstract model checking imprecise yet keeping the validity of the given formula.

In Section 4 , we shall show that the deformation of the Kripke structures we consider are induced by transformations of the source program, that act as an obfuscation strategy against an attack specified by an abstract model-checker. Accordingly, we say that an obfuscation is a transformation $\mathcal{O}: \mathcal{P} \rightarrow \mathcal{P}$ such that for a given formula $\phi$ and program $p \in \mathcal{P}: \mathcal{K}(p) \models \phi \Rightarrow \mathcal{K}(\mathcal{O}(p)) \models \phi$ and there exists a partition $P$ such that $\mathcal{K}(p)_{P} \models \phi \Rightarrow \mathcal{K}(\mathcal{O}(p))_{P} \not \models \phi$.

Example 4 (Model deformation). Consider the program $p$ from Example 2 The first step of refinement with the CEGAR algorithm with the initial partition (1) (described in Example 3) results in the partition (2). Intuitively, a deformation of the Kripke structure that forced the CEGAR algorithm to split the sets of variable values in classes smaller than the ones in partition (2) would weaken the power of CEGAR. To this aim, consider a deformation $\mathfrak{D}(\mathcal{K})$ of the concrete Kripke structure $\mathcal{K}$ of Example 3 obtained by duplicating $\mathcal{K}$ in such a way that one copy is kept isomorphic to the original one, while the second copy is modified by adding and removing some transitions to make the CEGAR algorithm less efficient. The copies can be obtained by introducing a new variable $z \in\{1,2\}$ : for $z=1$ we preserve all transitions, while for $z=2$ we change them to force a finer partition when a step of the CEGAR algorithm is applied. For example, in the replica for $z=2$, let us transform the copy of the dead state $(x=2, y=0, p c=2)$ into a bad state. This is obtained by adding and removing some transitions. After this transformation, assuming an initial partition analogous to partition (1),

$$
x:\{\{0\},\{1,2\}\} \quad y:\{\{2\},\{0,1,3,4,5\}\} \quad p c:\{\{1\},\{2,3,4,5\}\} \quad z:\{\{1,2\}\}
$$

where all the values of the new variable $z$ are kept together, we obtain an abstract Kripke structure isomorphic to the one of Fig. 3 with the same counterexamples. However, when we focus on the spurious counterexample, the situation is slightly changed. This is shown in Fig. 5, where the relevant point is the overall shape of the model and not the actual identity of each node. Roughly it combines two copies of the states in Fig. 4 those with $z=1$ are on the left and those with $z=2$ are on the right. The abstract state

$$
(x \in\{1,2\}, y \in\{0,1,3,4,5\}, p c \in\{2,3,4,5\}, z \in\{1,2\})
$$


is still a failure state, but it has three bad states and three dead states:

\begin{tabular}{|c|c|}
\hline bad states & dead states \\
\hline $\begin{array}{l}(x=1, y=1, p c=3, z=1) \\
(x=1, y=1, p c=3, z=2) \\
(x=2, y=0, p c=2, z=2)\end{array}$ & $\begin{array}{l}(x=1, y=0, p c=2, z=1) \\
(x=1, y=0, p c=2, z=2) \\
(x=2, y=0, p c=2, z=1)\end{array}$ \\
\hline
\end{tabular}

The bad state $(x=2, y=0, p c=2, z=2)$ and the dead states $(x=1, y=$ $0, p c=2, z=2)$ and $(x=1, y=4, p c=3, z=1)$ are incompatible. Therefore, the refinement leads to the partition below, where all values of $x$ are separated:

$x:\{\{0\},\{1\},\{2\}\} z:\{\{1\},\{2\}\} y:\{\{2\},\{0\},\{1,3,4,5\}\} p c:\{\{1\},\{2\},\{3,4,5\}\}$

\subsection{Measuring Obfuscations}

Intuitively, the largest is the size of the abstract Kripke structure to be model checked without spurious counterexamples, the harder is for the attacker to reach its goal. The interesting case is of course when the property $\phi$ holds true, but the abstraction used by the attacker leads to spurious counterexamples.

We propose to measure and compare obfuscations on the basis of the size of the final abstract Kripke structure where the property can be directly proved. As the abstract states are generated by a partition of the domains of each variable, the size is obtained just as the product of the number of partition classes for each variable. As obfuscations can introduce any number of additional variables over arbitrary domains, we consider only the size induced by the variables in the original program (otherwise increasing the number of variables could increase the measure of obfuscation without necessarily making CEGAR ineffective).

In the following we assume that $p$ is a program with variables $X$ and variables of interest $Y \subseteq X$ and $\phi$ is the formula that the attacker wants to prove.

Definition 1 (Size of a partition). Given a partition $P$ of $X$, we define the size of $P$ w.r.t. $Y$ as the natural number $\prod_{y \in Y}|P(y)|$.

Definition 2 (Measure of obfuscation). Let $\mathcal{O}(p)$ be an obfuscated program. The measure of $\mathcal{O}(p)$ w.r.t. $\phi$ and $Y$, written $\#_{\phi}^{Y} \mathcal{O}(p)$, is the size of the final partition $P$ w.r.t. $Y$ as computed by the above model of the attacker.

Our definition is parametric w.r.t. to the heuristics implemented in check (choice of the counterexample) and refine (how to partition irrelevant states). There are two main reasons for which the above measure is more significant than other choices, like counting the number of refinements: i) it is independent from the order in which spurious counterexamples are eliminated; ii) since the attacker has limited resources, a good obfuscation is the one that forces the attacker to model check a Kripke structure as large as possible.

Definition 3 (Comparing obfuscations). The obfuscated program $\mathcal{O}_{1}(p)$ is as good as $\mathcal{O}_{2}(p)$, written $\mathcal{O}_{1}(p) \geq_{\phi}^{Y} \mathcal{O}_{2}(p)$, if $\#_{\phi}^{Y} \mathcal{O}_{1}(p) \geq \#_{\phi}^{Y} \mathcal{O}_{2}(p)$. 


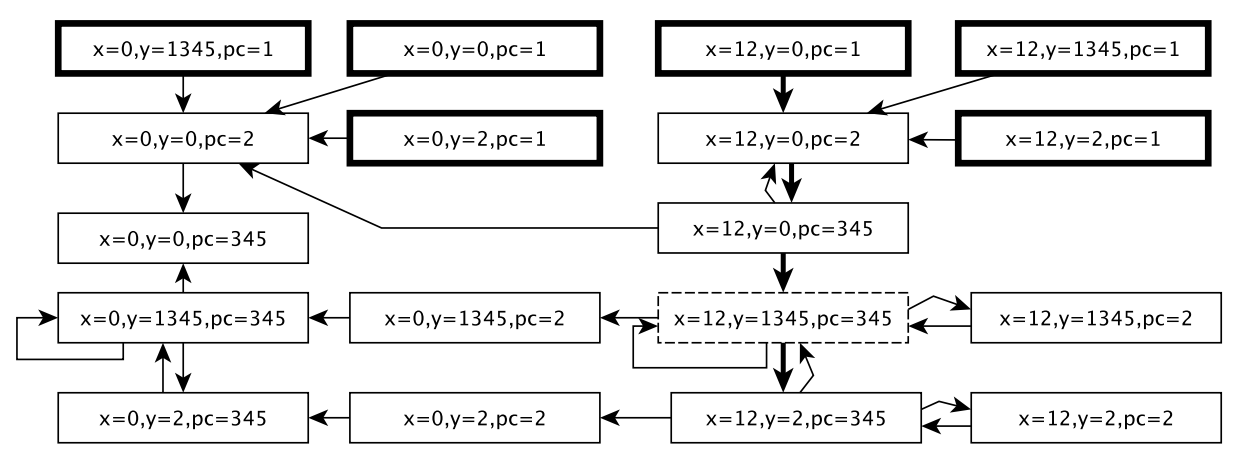

Fig. 6. A refined abstract Kripke structure

It follows that the best measure associated with an obfuscation is $\prod_{y \in Y}\left|D_{y}\right|$, where $D_{y}$ denotes the domain of $y$. This is the case where the abstraction separates all the concrete values that the variables in $Y$ may assume.

Example 5 (Ctd.). Let us consider again the running example and compare it with the semantically equivalent program $p^{\prime}$ below:

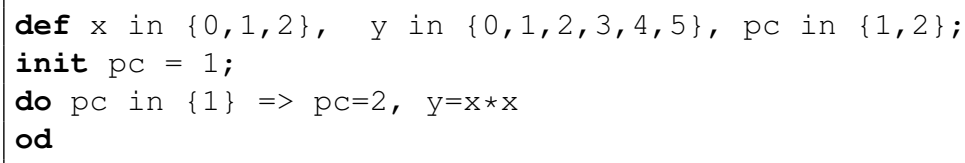

Let $x, y$ be the variables of interest. We have $\#_{\phi}^{\{x, y\}} p^{\prime}=2$, because the initial partition (1) is sufficient to prove that the property $\phi$ holds.

For the obfuscated program $p$, the size of the initial partition is just 4 (see partition (1) and the corresponding abstract Kripke structure in Fig. 3), and after one step of the CEGAR refinement the size of the computed partition is 6 (see partition (2) and the corresponding Kripke structure in Fig. 6). Since spurious counterexamples are still present, one more step of refinement is needed. When the attacker executes the procedure on the failure state marked with dashed border in Fig. 6, the result is the partition

$x:\{\{0\},\{1\},\{2\}\} \quad y:\{\{0\},\{1\},\{2\},\{3\},\{4,5\}\} \quad p c:\{\{1\},\{2\},\{4\},\{3,5\}\}$

whose size is 15 as it has been necessary to split all values for $x$ and most values for $y$. Now no more (spurious) counterexample can be found because all the states that invalidate the property are not reachable from initial states. Thus $\#_{\phi}^{\{x, y\}} p=15$, while the best obfuscation would have measure 18 , which is the product of the sizes of the domains of $x$ and $y$. As the reader may expect, we conclude that $p \geq_{\phi}^{\{x, y\}} p^{\prime}$. In our running example, for simplicity, we have exploited a very small domain for each variable, but if we take larger domains of values, then $\#_{\phi}^{\{x, y\}} p^{\prime}$ and $\#_{\phi}^{\{x, y\}} p$ remain unchanged, while the measure of the best possible obfuscation would grow considerably. 


\section{Best Code Obfuscation Strategy}

In the following, given an abstract Kripke structure $K$ and a property $\phi$, we let $S_{\phi}$ denote the set of abstract states that contain only concrete states that satisfy $\phi$, and $S_{\bar{\phi}}$ be the set with at least one concrete state that does not satisfy $\phi$. We denote by $\overline{\mathcal{O}}_{\phi}$ the obfuscation strategy realized by the following algorithm.

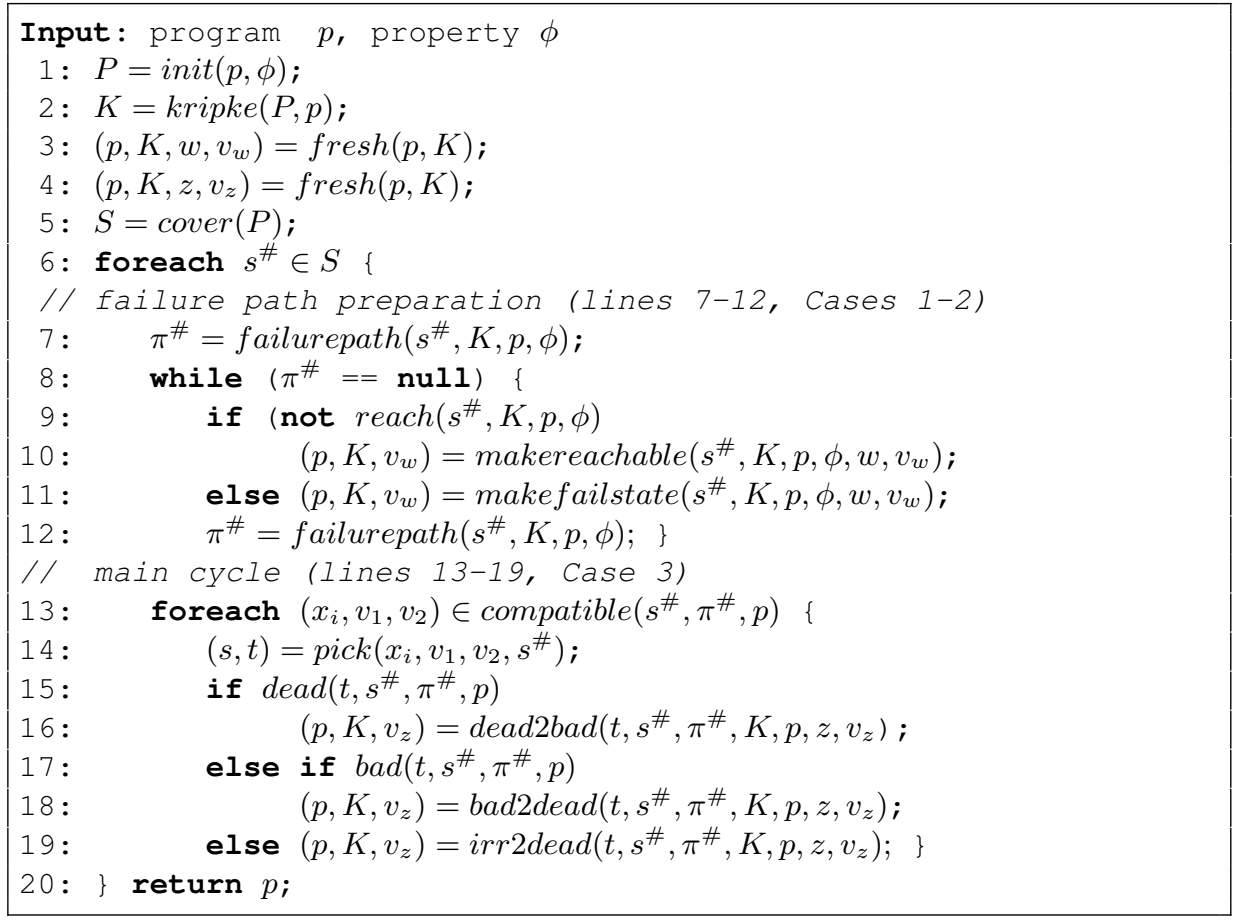

The algorithm starts by computing an initial partition $P$ and the corresponding abstract Kripke structure $K$. We want to modify the concrete Kripke structure so that CEGAR will split the abstract states in trivial partition classes for the variables of interest. The idea is to create several replicas of the concrete Kripke structure, such that one copy is preserved while the others will be changed by introducing and deleting arcs. This is obtained by introducing a new variable $z$ over a suitable domain $D_{z}=\{1, \ldots, n\}$ such that the concrete Kripke structure is replicated for each value $z$ can take. As a matter of notation, we denote by $(s, z=v)$ the copy of the concrete state $s$ where $z=v$. Without loss of generality, we assume that for $z=1$ we keep the original concrete Kripke structure. In practice such value of $z$ is hidden by an opaque expression. Actually we use two fresh variables, named $w$ and $z$ (lines 3 and 4 ): the former is used to introduce spurious counterexamples and failure states in the replica and the latter to force the splitting of failure states into trivial partition classes. The function fresh updates the program $p$ and the Kripke structure $K$ by taking into account the new variables and initializes the variables $v_{w}$ and $v_{z}$ that keep track of the last used values for $w$ and $z$. When a new replica is created, such values are incremented. 
The function cover (at line 5) takes the initial partition $P$ and returns a set of abstract states $s_{1}^{\#}, \ldots, s_{k}^{\#}$, called a covering, such that, together, they cover all non-trivia ${ }^{4}$ partition classes of the domains of the variable of interest, i.e. for each variable $x_{i}$, with $i \in[1, n]$, for each class $C \in P\left(x_{i}\right)$ such that $|C|>1$ there is an abstract state $s_{j}^{\#}$ with $j \in[1, k]$ and a concrete state $s \in s_{j}^{\#}$ such that $s\left(x_{i}\right) \in C$. Note that the set of all abstract states is a (redundant) covering.

For each $s^{\#} \in\left\{s_{1}^{\#}, \ldots, s_{k}^{\#}\right\}$ in the covering, there are three possibilities:

1. $s^{\#}$ does not contain any concrete state that is reachable via a concrete path that traverses only abstract states in $S_{\phi}$;

2. $s^{\#}$ is not a failure state but it contains at least one concrete state that is reachable via a concrete path that traverses only abstract states in $S_{\phi}$;

3. $s^{\#}$ is already the failure state of a spurious counterexample.

In case (3), failurepath $\left(s^{\#}, K, p, \phi\right)$ (line 7$)$ returns an abstract path that is a counterexample for $\phi$, in the other cases the function failurepath $\left(s^{\#}, K, p, \phi\right)$ returns null and the algorithm enters a cycle (to be executed at most twice, see lines 8-12) that transforms the Kripke structure and the program to move from cases (1-2) back to case (3), in a way that we will explain later.

Case (3) (lines 13-19). The core of the algorithm applies to a failure state $s^{\#}$ of a spurious counterexample $\pi^{\#}$. In this case the obfuscation method will force CEGAR to split the failure state $s^{\#}$ by separating all values in the domains of the variables of interest. Remember that CEGAR classifies the concrete states in $s^{\#}$ in three classes (bad, dead and irrelevant) and that dead states cannot be merged with bad or irrelevant states. We say that two states that can be merged are compatible. The role of the new copies of the Kripke structure is to prevent any merge between concrete states in the set $s^{\#}$. This is done by making sure that whenever two concrete states $(s, z=1),(t, z=1) \in s^{\#}$ can be merged into the same partition, then the states $\left(s, z=v_{z}+1\right)$ and $\left(t, z=v_{z}+1\right)$ cannot be merged together, because one is dead and the other is bad or irrelevant.

The function compatible $\left(s^{\#}, \pi^{\#}, p\right)$ returns the set of triples $\left(x_{i},\left\{v_{1}, v_{2}\right\}\right)$ such that $x_{i}$ is a variable of interest and any pair of states $\left(s, x_{i}=v_{1}\right),\left(s, x_{i}=v_{2}\right) \in$ $s^{\#}$ that differ just for the value of $x_{i}$ are compatible. Thus, the cycle at line 13 considers all such triples to make them incompatible. At line 14, we pick any two compatible states $(s, z=1)$ and $(t, z=1)$ such that $s\left(x_{i}\right)=v_{1}, t\left(x_{i}\right)=v_{2}$ and $s(x)=t(x)$ for any variable $x \neq x_{i}$. Given the spurious counterexample $\pi^{\#}$ with failure state $s^{\#}$ and a concrete state $t \in s^{\#}$ for the program $p$, the predicate $\operatorname{dead}(\cdot)$ returns true if the state $(t, z=1)$ is dead (line 15). Similarly, the predicate $b a d(\cdot)$ returns true if the state $(t, z=1)$ is bad (line 17$)$.

If $(t, z=1)$ is dead (w.r.t. $s^{\#}$ and $\left.\pi^{\#}\right)$, then it means that $(s, z=1)$ is also dead (because they are compatible), so we apply a dead-to-bad transformation to $t$ in the replica for $z=v_{z}+1$. This is achieved by invoking the function $\operatorname{dead2bad}(\cdot)$ (line 16) to be described below. The transformations bad2dead $(\cdot)$ (line 18) and $\operatorname{irr} 2 d e a d(\cdot)$ (line 19) apply to the other classifications for $(t, z=1)$.

\footnotetext{
${ }^{4}$ A partition class is trivial if it contains only one value.
} 
In the following, given a concrete state $s=\left(x_{1}=w_{1}, \ldots, x_{n}=w_{n}\right)$, we denote by $G(s)$ the guard $x_{1} \in\left\{w_{1}\right\} \wedge \ldots \wedge x_{n} \in\left\{w_{n}\right\}$ and by $A(s)$ the assignment $x_{1}=w_{1}, \ldots, x_{n}=w_{n}$. Without loss of generality, we assume for brevity that the abstract counterexample is formed by the abstract path prefix $\pi^{\#}=\left\{s_{0}^{\#}, s_{1}^{\#}, s^{\#}, s_{2}^{\#}\right\}$, with $s^{\#}$ the failure state and $t$ is the concrete state in $s^{\#}$ that we want to transform (see Figs. 77 9).

dead2bad $(\cdot)$. To make $t$ bad, the function must remove all concrete paths to $t$ along the abstract counterexample $\pi^{\#}$ and add one arc from $t$ to some concrete state $t^{\prime}$ in $s_{2}^{\#}$. To remove all concrete paths it is enough to remove the arcs from states in $s_{1}^{\#}$ to $t$ (see Fig. 7). At the code level, dead2bad $(\cdot)$ modifies each command $g \Rightarrow a$ such that there is some $s^{\prime} \in s_{1}^{\#}$ with $s^{\prime} \models g$ and $t=s^{\prime}[a]$. Given $t$ and $s_{1}^{\#}$, let $S(g \Rightarrow a)=\left\{s^{\prime} \in s_{1}^{\#} \mid s^{\prime} \models g \wedge t=s[a]\right\}$. Each command $c=(g \Rightarrow a)$ such that $S(c) \neq \emptyset$ is changed to the command

$$
g \wedge\left(z \notin\left\{v_{z}+1\right\} \vee \bigwedge_{s^{\prime} \in S(c)} \neg G\left(s^{\prime}\right)\right) \Rightarrow a .
$$

When $z \neq v_{z}+1$ the updated command is applicable whenever $c$ was applicable. When $z=v_{z}+1$ the command is not applicable to $z=v z+1$ the states in $S(c)$. To add the arc from $t$ to a state $t^{\prime}$ in $s_{2}^{\#}$ it is enough to add the command $z \in\left\{v_{z}+1\right\} \wedge G(t) \Rightarrow A\left(t^{\prime}\right)$.

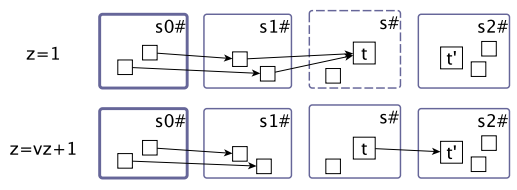

Fig. 7. From dead to bad

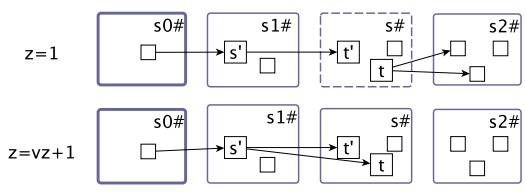

Fig. 8. From bad to dead $z=1$

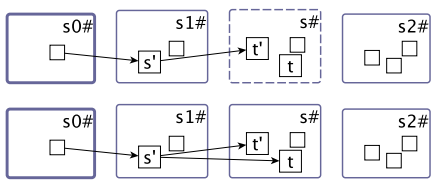

Fig. 9. From irrelevant to dead bad2dead $(\cdot)$. The function selects a dead state $t^{\prime}$ in the failure state $s^{\#}$ and a concrete path $\pi=\left\langle s_{0}, s^{\prime}, t^{\prime}\right\rangle$ to $t^{\prime}$ along the abstract counterexample $\pi^{\#}$. To make $t$ dead in the replica with $z=v_{z}+1$, the function adds a concrete arc from $s^{\prime}$ to $t$ and removes all arcs leaving from $t$ to concrete states in $s_{2}^{\#}$ (see Fig. 8). To insert the arc from $s^{\prime}$ to $t$, the command $G\left(s^{\prime}\right) \wedge z \in\left\{v_{z}+1\right\} \Rightarrow A(t)$ is added. To remove all arcs leaving from $t$ to concrete states in $s_{2}^{\#}$, the function changes the guard $g$ of each command $g \Rightarrow a$ such that $t=g$ and $t[a] \in s_{2}^{\#}$ to $g \wedge\left(z \notin\left\{v_{z}+1\right\} \vee \neg G(t)\right)$, which is applicable to all the states different from $t$ where $g \Rightarrow a$ was applicable as well as to the replicas of $t$ for $z \neq v_{z}+1$.

$\operatorname{irr} 2 \operatorname{dead}(\cdot)$. Here the state $t$ is irrelevant and we want to make it dead. The function builds a concrete path to $t$ along the abstract counterexample $\pi^{\#}$. As before, it selects a dead state $t^{\prime}$ in the failure state and a concrete path $\pi=\left\langle s_{0}, s^{\prime}, t^{\prime}\right\rangle$ to $s$ along the abstract counterexample $\left\langle s_{0}^{\#}, s_{1}^{\#}, s^{\#}\right\rangle$. To make $t$ dead the function adds an arc from $s^{\prime}$ (i.e., the state that immediately precedes $t^{\prime}$ in $\pi$ ) to $t$ (see Fig. 9). For the program it is sufficient to add a new command with guard $G\left(s^{\prime}\right) \wedge z \in\left\{v_{z}+1\right\}$ and whose assignment is $A(t)$. 
From cases (1-2) to case (3) (lines 7-12). The predicate reach $\left(s^{\#}, K, p, \phi\right)$ is true if we are in case (2) and false if we are in case (1). In both cases we apply some preliminary transformations to $K$ and $p$ after which $s^{\#}$ is brought to case (3). The function makereachable $\left(s^{\#}, K, p, \phi, w, v_{w}\right)$ transforms the Kripke structure so that in the end $s^{\#}$ contains at least one concrete state that is reachable via a concrete path that traverses only abstract states in $S_{\phi}$, moving from case (1) to cases (2) or (3), while the function makefailstate $\left(s^{\#}, K, p, \phi, w, v_{w}\right)$ takes $s^{\#}$ satisfying case (2) and it returns a modi-

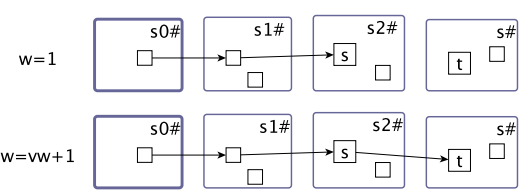

Fig. 10. Case 1: makereachable $(\cdot)$

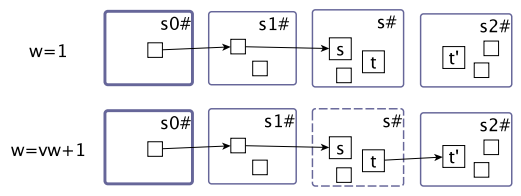

Fig. 11. Case 2: makefailstate $(\cdot)$ fied Kripke structure where $s^{\#}$ now falls in case (3). The deformations are illustrated in Figs. 10 11. At the code level, addition and removal of arcs is realized as detailed before.

\section{Main Results}

Our obfuscation preserves the semantics of the program. This is because all the transformations we have discussed maintain the original Kripke structure associated with a distinguished value of the new variables $w$ and $z$ that are introduced. Indeed, when the obfuscated program is executed with initial values $w=1$ and $z=1$ it behaves exactly as the original program. By exploiting opaque expressions to initialise the variables $w$ and $z$, we hide their values from the attacker who has to take into account all possible values for $w$ and $z$ and thus run CEGAR on the deformated Kripke structure.

Theorem 1 (Embedding). Let $p=(d ; g ; c)$ and $\overline{\mathcal{O}}_{\phi}(p)=\left(\left(d, w \in D_{w}, z \in\right.\right.$ $\left.\left.D_{z}\right) ; g ; c^{\prime}\right)$, then $\mathcal{K}(p)$ is isomorphic to $\mathcal{K}\left((d, w \in\{1\}, z \in\{1\}) ; g ; c^{\prime}\right)$.

The isomorphism at the level of Kripke structures guarantees that the obfuscation does not affect the number of steps required by any computation, i.e., to some extent the efficiency of the original program is also preserved.

Second, the obfuscation preserves the property $\phi$ of interest when the program is executed with any input data for $w$ and $z$, i.e. $\phi$ is valid in all replicas.

Theorem 2 (Soundness). $\mathcal{K}(p) \models \phi$ iff $\mathcal{K}\left(\overline{\mathcal{O}}_{\phi}(p)\right) \models \phi$.

Note that Theorem 1 guarantees that the semantics is preserved entirely, i.e. not only $\phi$ is preserved in all replicas (Theorem 2) but any other property is preserved when the obfuscated program is run with $w \in\{1\}$ and $z \in\{1\}$.

The next result guarantees the optimality of obfuscated programs.

Theorem 3 (Hardness). $\#_{\phi}^{Y} \overline{\mathcal{O}}_{\phi}(p)=\prod_{y \in Y}\left|D_{y}\right|$. 


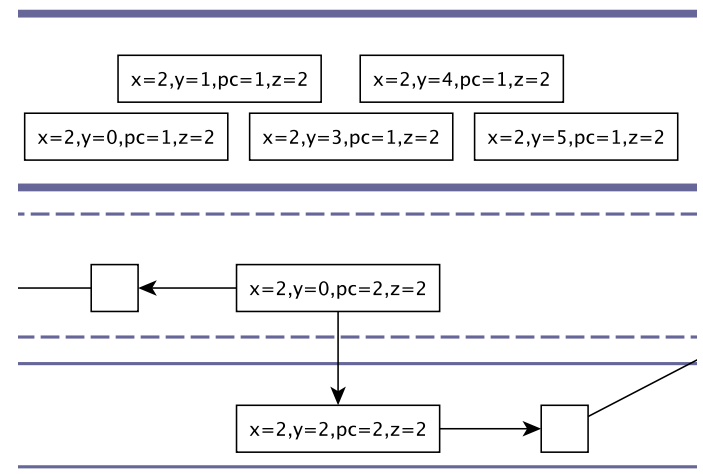

Fig. 12. A fragment of the model for $z=2$

As a consequence, for any program $p$ and formula $\phi$ the function mapping $\mathcal{K}(p)$ into $\mathcal{K}\left(\overline{\mathcal{O}}_{\phi}(p)\right)$ is a model deformation for all (non-trivial) partitions of the variables of interest.

Theorem 4 (Complexity). The complexity of our best code obfuscation strategy is polynomial in the size of the domains of the variables of interest $Y$.

Example 6 (Best code obfuscation). Consider the abstract Kripke structure in Fig. 3. The failure state $s^{\#}=(x \in\{1,2\}, y \in\{0,1,3,4,5\}, p c \in\{2,3,4,5\})$ covers all the non-trivial partition classes for the variables of interest $x$ and $y$. Since it is a failure state for an abstract counterexample, we are in case (3). For simplicity, since the transformations for cases (1-2) are not needed, we omit the insertion of the variable $w$.

The dead state $(x=1, y=0, p c=2)$ is incompatible with the irrelevant state $(x=1, y=1, p c=2)$, thus the triple $(y,\{1,2\})$ is incompatible. For the same reason the value 0 for $y$ is also separated from the values $3,4,5$. Our obfuscation must separate the values 1,2 for $x$ and the values $1,3,4,5$ for $y$. Therefore at most 6 replicas are needed. In the end, 5 values for $z$ suffices. Let us take the triple $(x,\{1,2\})$ and let us pick the two dead states $t=(x=2, y=0, p c=2)$ and $s=(x=1, y=0, p c=2)$ in Fig. 4 The algorithm invokes bad2dead $(\cdot)$ on state $(t, z=2)$ to make it incompatible with the dead state $(x=1, y=0, p c=$ $2, z=2$ ). At the code level, we note that all incoming arcs of $t$ are due to the command $c_{1}$ (see Fig. 4). To remove them, $c_{1}$ becomes $c_{1,1}$.

$\mathrm{pc}$ in $\{1\} /(\mathrm{z}$ notin $\{2\} \backslash / \mathrm{x} \operatorname{notin}\{2\} \backslash / \mathrm{y} \operatorname{notin}\{2\}) \Rightarrow \mathrm{pc}=2, \mathrm{y}=0 \quad \% \mathrm{c} 11$

Moreover, to make $(t, z=2)$ a bad state, is added an arc from the state $(t, z=2)$, to $(x=2, y=2, p c=2, z=2)$ with the new command $c_{1,2}$

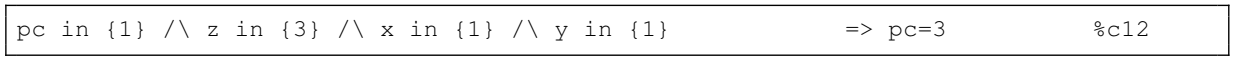

In Fig. 12 we show the relevant changes on the Kripke structure for the replica with $z=2$ (compare it with Fig. 4). To complete the obfuscation more transformations are required: one bad-to-dead and two irrelevant-to-dead transformations. Finally, we obtain the program $p o=\overline{\mathcal{O}}_{\phi}(p)$ below: 


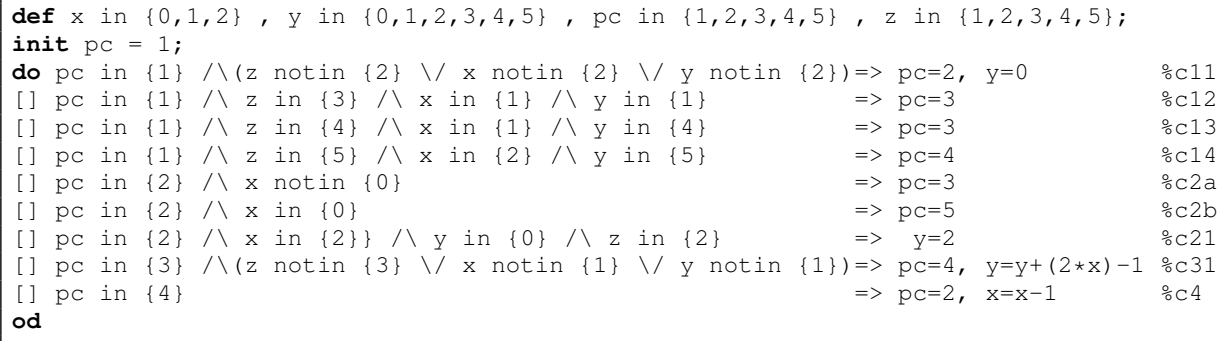

Let us assume that the attacker starts with the abstraction of the obfuscated program induced by the partition $x:\{\{0\},\{1,2\}\}, y:\{\{2\},\{0,1,3,4,5\}\}$, $p c:\{\{1\},\{2,3,4,5\}\}$, and $z:\{\{1,2,3,4,5\}\}$. The abstract Kripke structure is isomorphic to the one in Fig. 3 having several spurious counterexamples for $\phi$. One such path is similar to the one in Fig. $3 .\left\{s_{0}^{\#}, s_{1}^{\#}, s_{2}^{\#}\right\}$ with:

$$
\begin{aligned}
& s_{0}^{\#}=x \in\{1,2\}, y \in\{0,1,3,4,5\}, p c \in\{1\}, z \in\{1,2,3,4,5\} \\
& s_{1}^{\#}=x \in\{1,2\}, y \in\{0,1,3,4,5\}, p c \in\{2,3,4,5\}, z \in\{1,2,3,4,5\} \\
& s_{2}^{\#}=x \in\{1,2\}, y \in\{2\}, p c \in\{2,3,4,5\}, z \in\{1,2,3,4,5\} .
\end{aligned}
$$

The failure state is $s_{1}^{\#}$. It has 5 bad concrete states and 12 dead states. By CEGAR we get the partition that has only trivial (singletons) classes. Therefore the abstract Kripke structure coincides with the concrete Kripke structure: it has 450 states of which 90 are initial states.

Given that the variables of interest are $x$ and $y$, the measure of the obfuscation is 18, i.e., it has the maximum value and thus $p o \geq_{\phi}^{\{x, y\}} p \geq_{\phi}^{\{x, y\}} p^{\prime}$. We remark that when $z=1$, po has the same semantics as $p$ and $p^{\prime}$.

The guarded command po can be understood as a low-level, flattened description for programs written in any language. However, it is not difficult to derive, e.g., an ordinary imperative program from a given guarded command. We do so for the reader's convenience.

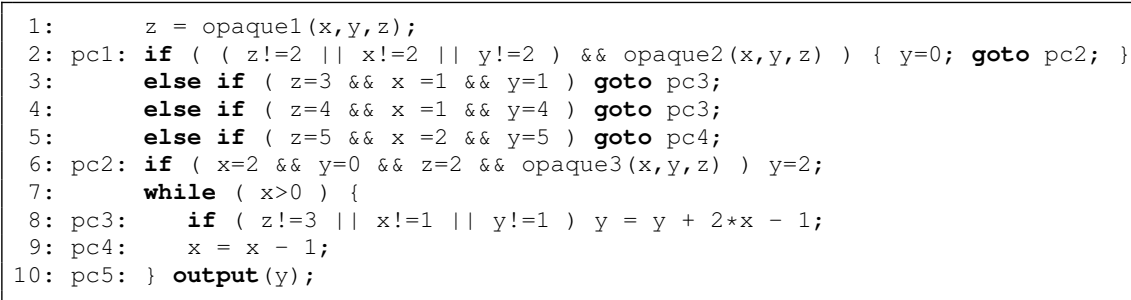

To hide the real value of $z$ we initialise the variable using an opaque expression opaque $1(x, y, z)$ whose value is 1 . Moreover, one has to pay attention to the possible sources of nondeterminism, which can arise when there are two or more guarded commands $g_{1} \Rightarrow a_{1}$ and $g_{2} \Rightarrow a_{2}$ and a state $s$ such that $s \models g_{1}$ and $s \models g_{2}$. The idea is to introduce opaque predicates so that the exact conditions under which a branch is taken are hard to determine by the attacker, who has to take into account both possibility (true and false) as a nondeterministic choice. 
In our example, the sources of nondeterminism are due to the pairs of commands $\left(c_{1,1}, c_{1,2}\right),\left(c_{1,1}, c_{1,3}\right),\left(c_{1,1}, c_{1,4}\right)$ and $\left(c_{2,1}, c_{2 a}\right)$. Consequently, we assume two opaque predicates opaque $2(x, y, z)$ and opaque $3(x, y, z)$ are available. In order to preserve the semantics, for $z=1$ we require that opaque $1(x, y, z)$ returns true, while opaque $2(x, y, z)$ is unconstrained. Finally, since the program counter is an explicit variable in guarded commands, we represent its possible values by labels and use goto instructions accordingly. Thus we write the label pcn to denote states where $p c=n$ and write goto $p c n$ for assignments of the form $p c=n$.

\section{Discussion}

We have shown that it is possible to systematically transform Kripke structures in order to make automated abstraction refinement by CEGAR hard. Addressing refinement procedures instead of specific abstractions makes our approach independent from the chosen abstraction in the attack.

To enforce the protection of the real values of variable $w$ (and analogously for $z$ ) initialized by opaque functions against more powerful attacks able to inspect the memory of different program runs, one idea is to use a class of values instead of a single value. This allows the obfuscated code to introduce instructions that assign to $w$ different values in the same class, thus convincing the attacker that the value of $w$ is not invariant.

The complexity of our best code obfuscation strategy is polynomial in the size of the domains of the variables of interest. Moreover, we note that the same algorithm can produce a valuable obfuscation even if one selects a partial cover instead of a complete one: in this case, it is still guaranteed that the refinement strategy will be forced to split all the values appearing in the partial cover. This allows to choose the right trade-off between the complexity of the obfuscation strategy and the measure of the obfuscated program. It is also possible that the algorithm introduces more transformations than strictly necessary. This is because the obfuscation is performed w.r.t. an initial partition. To further reduce the number of transformations, we can apply the obfuscation only at the end of the abstract model checking process, where less pairs of values needs to be separated. Of course, this strategy would not influence the measure of the result.

As already mentioned, our obfuscation assumes that CEGAR makes dead states incompatible with both irrelevant and bad states. Our algorithm can be generalised to the more general setting where dead states are only incompatible with bad states. Therefore even if the attacker had the power to compute the coarsest partition that separates bad states from dead states (which is a NP-hard problem) our strategy would force the partition to consist of trivial classes only.

We can see abstraction refinement as a learning procedure which learns the coarsest state equivalence by model checking a temporal formula. Our results provide a very first attempt to defeat this procedure.

As an ongoing work, we have extended our approach to address attacks aimed to disclose data-flow properties and watermarks. It remains to be investigated 
how big the text of the best obfuscated program can grow: limiting its size is especially important in the case of embedded systems.

We plan to extend our approach to other abstraction refinements, like predicate refinement and the completeness refinement in [16] for generic abstract interpreters and more in general for a machine learning algorithm. This would make automated reverse engineering hard in more general attack models.

Acknowledgement. We are very grateful to Alberto Lluch-Lafuente for the fruitful discussions we had on the subject of this paper.

\section{References}

1. S. Banescu, C. S. Collberg, V. Ganesh, Z. Newsham, and A. Pretschner. Code obfuscation against symbolic execution attacks. In S. Schwab, W. K. Robertson, and D. Balzarotti, editors, Proc. 32nd Annual Conference on Computer Security Applications, ACSAC 2016, pages 189-200. ACM, 2016.

2. E. Clarke, O. Grumberg, S. Jha, Y. Lu, and H. Veith. Counterexample-guided abstraction refinement for symbolic model checking. J. ACM, 50(5):752-794, 2003.

3. E. Clarke, O. Grumberg, and D. Long. Model checking and abstraction. In Proc. of the 19th ACM Symp. on Principles of Programming Languages (POPL '92), pages 343-354. ACM Press, 1992.

4. E. Clarke, O. Grumberg, and D. Long. Model checking and abstraction. ACM Trans. Program. Lang. Syst., 16(5):1512-1542, 1994.

5. E. Clarke, O. Grumberg, and D. Peled. Model Checking. The MIT Press, 1999.

6. C. Collberg, J. Davidson, R. Giacobazzi, Y. Gu, A. Herzberg, and F. Wang. Toward digital asset protection. IEEE Intelligent Systems, 26(6):8-13, 2011.

7. C. Collberg and J. Nagra. Surreptitious Software: Obfuscation, Watermarking, and Tamperproofing for Software Protection. Addison-Wesley Professional, 2009.

8. P. Cousot and R. Cousot. Abstract interpretation: A unified lattice model for static analysis of programs by construction or approximation of fixpoints. In Proc. of the 4th ACM Symp. on Principles of Programming Languages (POPL 'ry), pages 238-252. ACM Press, 1977.

9. P. Cousot and R. Cousot. An abstract interpretation-based framework for software watermarking. In Proc. of the 31st ACM Symp. on Principles of Programming Languages (POPL '04), pages 173-185. ACM Press, New York, NY, 2004.

10. M. Dalla Preda and R. Giacobazzi. Semantics-based code obfuscation by abstract interpretation. Journal of Computer Security, 17(6):855-908, 2009.

11. D. Dams, R. Gerth, and O. Grumberg. Abstract interpretation of reactive systems. ACM Trans. Program. Lang. Syst., 19(2):253-291, 1997.

12. E. A. Emerson. Temporal and modal logic. In J. van Leeuwen, editor, Handbook of Theoretical Computer Science, volume B: Formal Models and Semantics. Elsevier, Amsterdam and The MIT Press, Cambridge, Mass., 1990.

13. R. Giacobazzi. Hiding information in completeness holes - new perspectives in code obfuscation and watermarking. In Proc. of the 6th IEEE Int. Conferences on Software Engineering and Formal Methods (SEFM '08), pages 7-20. IEEE Press, 2008.

14. R. Giacobazzi, N. D. Jones, and I. Mastroeni. Obfuscation by partial evaluation of distorted interpreters. In Proc. of the ACM SIGPLAN Symp. on Partial Evaluation and Semantics-Based Program Manipulation (PEPM'12), pages 63-72. ACM Press, 2012. 
15. R. Giacobazzi and E. Quintarelli. Incompleteness, counterexamples and refinements in abstract model-checking. In Proc. of the 8th Int. Static Analysis Symp. (SAS'01), volume 2126 of Lecture Notes in Computer Science, pages 356-373. Springer, 2001.

16. R. Giacobazzi, F. Ranzato, and F. Scozzari. Making abstract interpretation complete. Journal of the ACM, 47(2):361-416, March 2000.

17. Microsoft. Static driver verifier website (last consulted november 2017), 2017. https://docs.microsoft.com/en-us/windows-hardware/drivers/ devtest/static-driver-verifier.

18. J. Nagra, C. D. Thomborson, and C. Collberg. A functional taxonomy for software watermarking. Aust. Comput. Sci. Commun., 24(1):177-186, 2002.

19. F. Ranzato and F. Tapparo. Generalized strong preservation by abstract interpretation. Journal of Logic and Computation, 17(1):157-197, 2007.

20. D. A. Schmidt. Data flow analysis is model checking of abstract interpretations. In D. B. MacQueen and L. Cardelli, editors, POPL '98, Proceedings of the 25th ACM SIGPLAN-SIGACT Symposium on Principles of Programming Languages, San Diego, CA, USA, January 19-21, 1998, pages 38-48. ACM, 1998.

21. D. A. Schmidt and B. Steffen. Program analysis as model checking of abstract interpretations. In G. Levi, editor, Static Analysis, 5th International Symposium, SAS '98, Pisa, Italy, September 14-16, 1998, Proceedings, volume 1503 of Lecture Notes in Computer Science, pages 351-380. Springer, 1998.

22. R. Venkatesan, V. Vazirani, and S. Sinha. A graph theoretic approach to software watermarking. In Proc. 4th Int. Workshop on Information Hiding (IHW '01), volume 2137 of Lecture Notes in Computer Science, pages 157-168. Springer, 2001.

23. C. Wang, J. Hill, J. C. Knight, and J. W. Davidson. Protection of softwarebased survivability mechanisms. In 2001 International Conference on Dependable Systems and Networks (DSN 2001) (formerly: FTCS), 1-4 July 2001, Göteborg, Sweden, Proceedings, pages 193-202. IEEE Computer Society, 2001. 